\title{
Variations physiologiques \\ de l'activité lipoprotéine-lipasique du lait de chèvre
}

\author{
par \\ Y. CHILLIARD et P. MORAND-FEHR \\ (avec la collaboration technique de M. DORLEANS) \\ Laboratoire de Recherches de la Chaire de Zootechnie (I.N.R.A.) \\ Institut National Agronomique Paris-Grignon \\ 16, rue Claude-Bernard - 75231 Paris cedex 05
}

\section{INTRODUCTION}

La lipolyse dans les produits laitiers, en particulier dans le lait refroidi, a pris une grande importance en technologie laitière comme en témoignent les rapports récents de la Fédération Internationale de Laiterie $(1974,1975)$.

Certains types de lipolyse (lipolyse "induite » et "spontanée ") résultent de l'action d'une (ou de plusieurs) lipase(s) sécrétée(s) dans le lait. Tarassuk et Frankel (1957) ont suggéré l'existence de plusieurs lipases distinctes responsables de la lipolyse dans le lait de vache. En 1962, Korn montra la présence d'une activité lipoprotéine-lipasique (LPL) dans ce lait. Les investigations récentes indiquent que l'activité lipolytique du lait de vache est due essentiellement à une seule enzyme (Hoynes et Downey, 1973 ; Castberg et al., 1975) ayant les caractéristiques des LPL tissulaires (Olivecrona et al., 1975). Toutefois, la corrélation entre l'activité LPL (ou " lipasique ") et la lipolyse spontanée est généralement faible (Dunkley et Smith, 1951 ; Driessen et Stadhouders, 1974 ; Castberg et Solberg, 1974). En effet, Deeth et Fitz-Gerald (1975) soulignent que la lipolyse résulte, en plus de l'activité de la (ou des) lipase(s), de la susceptibilité des globules gras à l'attaque de celle(s)-ci et de la présence ou de l'absence de facteurs inhibiteurs ou activateurs dans le lait de vache.

Par contre, dans le lait de femme, et alors que celui-ci contient une autre lipase très active et stimulée par les sels biliaires (Hernell et Olivecrona, 1974 a, b), l'activité LPL est en bonne corrélation avec la lipolyse (Luzeau et al., 1975 ; Castberg et Hernell, 1975). 
Compte tenu de ces variations suivant les espèces, il est nécessaire d'étudier les systèmes lipolytiques des laits des différentes espèces, utilisés en technologie laitière. Ainsi, chez la chèvre, le lait conservé à $4^{\circ} \mathrm{C}$ peut aussi présenter une lipolyse spontanée intense (Chilliard et Luzeau, 1975 ; Bjørke et Castberg, 1976). Ce lait contient une activité LPL (Chilliard et Fehr, 1976 a) qui semble être la seule activité lipasique importante (comme le lait de vache) et qui est bien corrélée avec l'intensité de la lipolyse spontanée (comme le lait de femme) (Bjørke et Castberg, 1976). Les variations physiologiques de l'activité LPL du lait de chèvre ont été peu étudiées jusqu'ici (Bjørke et Castberg, 1976 ; Chilliard et Fehr, 1976 b). Des investigations complémentaires sont nécessaires en raison de l'importance de l'activité LPL pour la lipolyse spontanée dans le lait de chèvre, et du fait que les facteurs physiologiques déterminent de grandes variations de la lipolyse spontanée dans le lait de vache (Jellema et Schipper, 1975 ; Deeth et Fitz-Gerald, 1976).

Cinq facteurs de variation de l'activité LPL du lait de chèvre ont donc été étudiés :

- le stade de traite ;

- la succession des traites, ces deux facteurs permettant de mieux connaître l'effet des conditions de prélèvement ;

- le stade de lactation, facteur d'autant plus important à préciser chez la chèvre qu'il existe une liaison prononcée entre les dates de collecte du lait et les stades de lactation, en raison du caractère saisonnier de la production laitière ;

- la répétition d'une lactation à la suivante ;

- le jeûne et la réalimentation puisque, chez la vache, l'état nutritionnel semble influer sur l'intensité de la lipolyse (Jellema et Schipper, 1975 ; Deeth et Fitz-Gerald, 1976) et sur l'activité lipasique du lait (Borges, 1975).

\section{MATERIEL ET METHODES}

Les chèvres utilisées dans ces expériences sont de race Alpine, âgées de 2 à 8 ans, et reçoivent du foin de luzerne à volonté et un mélange d'aliments concentrés : orge, avoine, tourteau de soja, minéraux, vitamines $(600 \mathrm{~g} / \mathrm{j}+300 \mathrm{~g} / \mathrm{kg}$ de lait au-dessus d'une production de $2 \mathrm{~kg}$ ). Le lait est prélevé à la traite du soir et représentatif de l'ensemble de la traite, et aussitôt filtré et congelé à $-25^{\circ} \mathrm{C}$. La congélation n'entraîne pas de baisse décelable de l'activité lipolytique pendant 3 mois au moins, alors qu'une perte d'activité d'environ 10 p. 100 est enregistrée après 6 mois de conservation. 
L'activité LPL du lait entier, après dilution dans une solution de $\mathrm{NaCl}$ à 9 p. 1000, est mesurée selon une modification de la méthode d'Egelrud et Olivecrona (1972), décrite par Chilliard et Fehr (1976 a). L'enzyme, présente dans le lait, hydrolyse les triglycérides d'une émulsion commerciale d'huile de soja (Intralipide-Vitrum), préalablement "activés " par du sérum sanguin de chèvre, au cours d'une incubation dans un milieu tamponné $(\mathrm{pH} 8,3)$ contenant de l'albumine bovine et de l'héparine comme co-facteurs et dans des conditions de linéarité en fonction du temps et de la concentration en lait. La vitesse de libération des acides gras du substrat est déterminée par la méthode semi-automatique d'Antonis (1965). Chaque incubation est effectuée en double. La reproductibilité (intra et interlaboratoire) du dosage est d'environ $10 \mathrm{p} .100$, pour des laits ayant des activités LPL comprises entre 0 et $70 \mu \mathrm{Eq} \mathrm{AG} / \mathrm{h} / \mathrm{ml}$ (Chilliard et Fehr, $1976 \mathrm{a}$ ).

La nature LPL de l'activité lipolytique enregistrée a été vérifiée à l'aide de plusieurs critères : faible inhibition par le $\operatorname{NaF}(0,02 \mathrm{M})$, forte inhibition par le $\mathrm{NaCl}(0,85 \mathrm{M})$ et le sulfate de protamine (1 à $10 \mathrm{mg} / \mathrm{ml}$ ), très faible activité lorsque le substrat n'est pas " activé » par du sérum sanguin, $\mathrm{pH}$ optimum alcalin $(8,3$ à 9,0$)$ (Chilliard et Fehr, 1976 a).

\section{RESULTATS}

\section{Variations au cours de la traite}

Le lait est prélevé manuellement sur cinq chèvres, quatre en $14^{\mathrm{e}}$ semaine et une en $7^{\mathrm{e}}$ semaine de lactation. L'activité LPL mesurée dans les 100 derniers $\mathrm{ml}$ de la traite est très significativement supérieure à celle mesurée dans les 100 premiers $\mathrm{ml}$ (tab. 1). Toutefois, les variations individuelles sont à l'origine de 89 p. 100 de la dispersion des résultats, et chez une des chèvres l'activité LPL est identique pour les deux stades de traite.

\section{Variations d'une traite à l'autre}

\section{Expérience 1}

Le lait est prélevé mécaniquement au cours de deux traites consécutives ( $7 \mathrm{~h}$ le matin et $17 \mathrm{~h}$ le soir), chez deux chèvres. Les activités LPL sont de 33,2 $\mu \mathrm{Eq} \mathrm{AG/h/mI} \mathrm{le} \mathrm{soir} \mathrm{et} \mathrm{48,5} \mathrm{le} \mathrm{matin} \mathrm{pour}$ un animal et de 7,7 le soir et 3,4 le matin pour l'autre animal.

\section{Expérience 2}

Le lait est prélevé manuellement au cours de quatre traites consécutives, chez cinq chèvres. Les variations individuelles sont importantes (fig. 1). Au cours des trois premières traites, l'activité LPL moyenne reste constante du fait des évolutions opposées des diffé- 


\section{TABLEAU 1}

Influence du stade de traite sur l'activité LPL du lait de chèvre

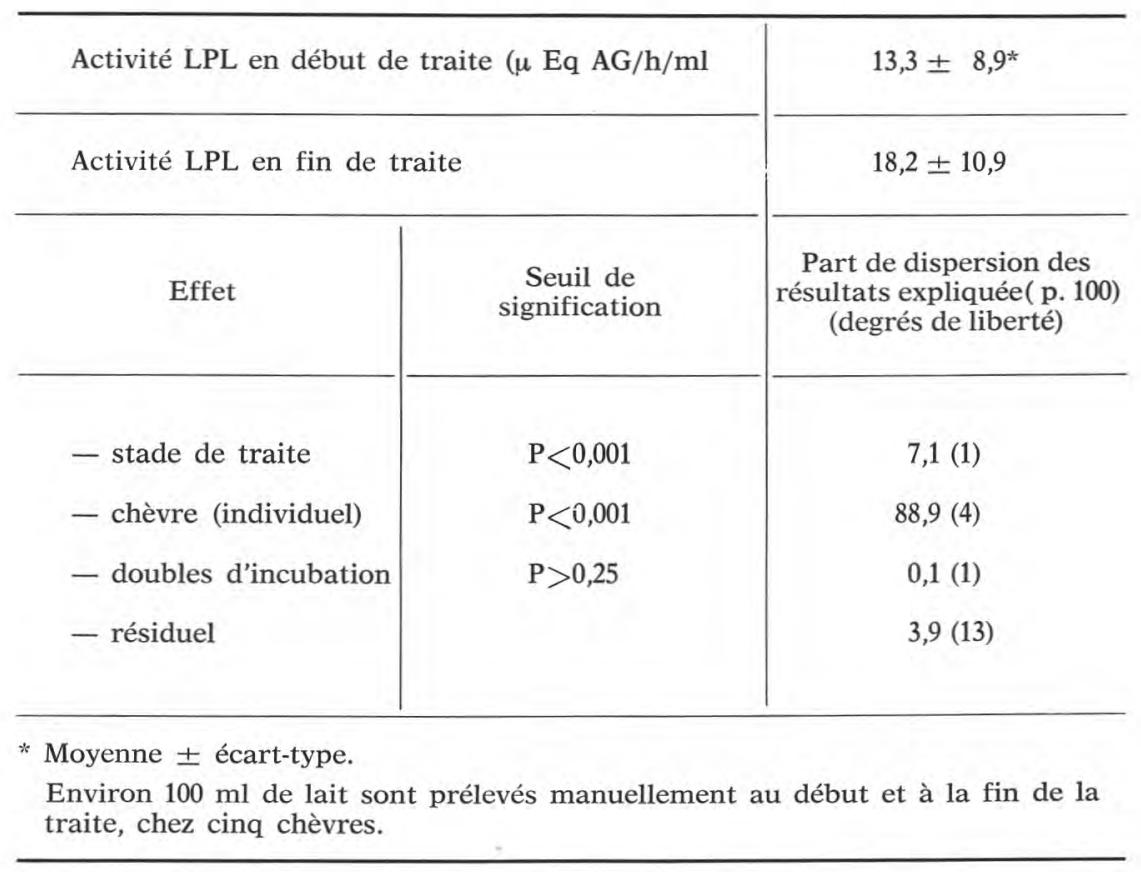

rentes chèvres, alors que l'activité LPL augmente fortement chez tous les animaux entre la troisième et la quatrième traite, d'où un effet très significatif des facteurs " jour » et " heure ", et de leur interaction.

\section{Variations au cours de la lactation}

Chez six chèvres, l'activité LPL du lait (prélevé mécaniquement) augmente fortement, en moyenne, jusqu'en $30^{\mathrm{e}}$ semaine et diminue brutalement après ce stade de lactation (fig. 2). Les activités LPL sont toutefois très variables d'une chèvre à l'autre pour un même stade de lactation et les évolutions dans le temps varient selon les animaux : une chèvre atteint l'activité LPL maximum en $14^{\mathrm{e}}$ semaine, deux en $22^{\mathrm{e}}$ semaine et trois en $30^{\mathrm{e}}$ semaine. L'analyse statistique des résultats montre que l'augmentation jusqu'en $30^{\mathrm{e}}$ semaine est très significative $(\mathrm{P}<0,001)$ et non significativement différente d'une augmentation linéaire. L'effet chèvre est aussi très significatif $(\mathrm{P}<0,001)$. L'activité LPL produite par jour (estimée en faisant 1'hypo- 


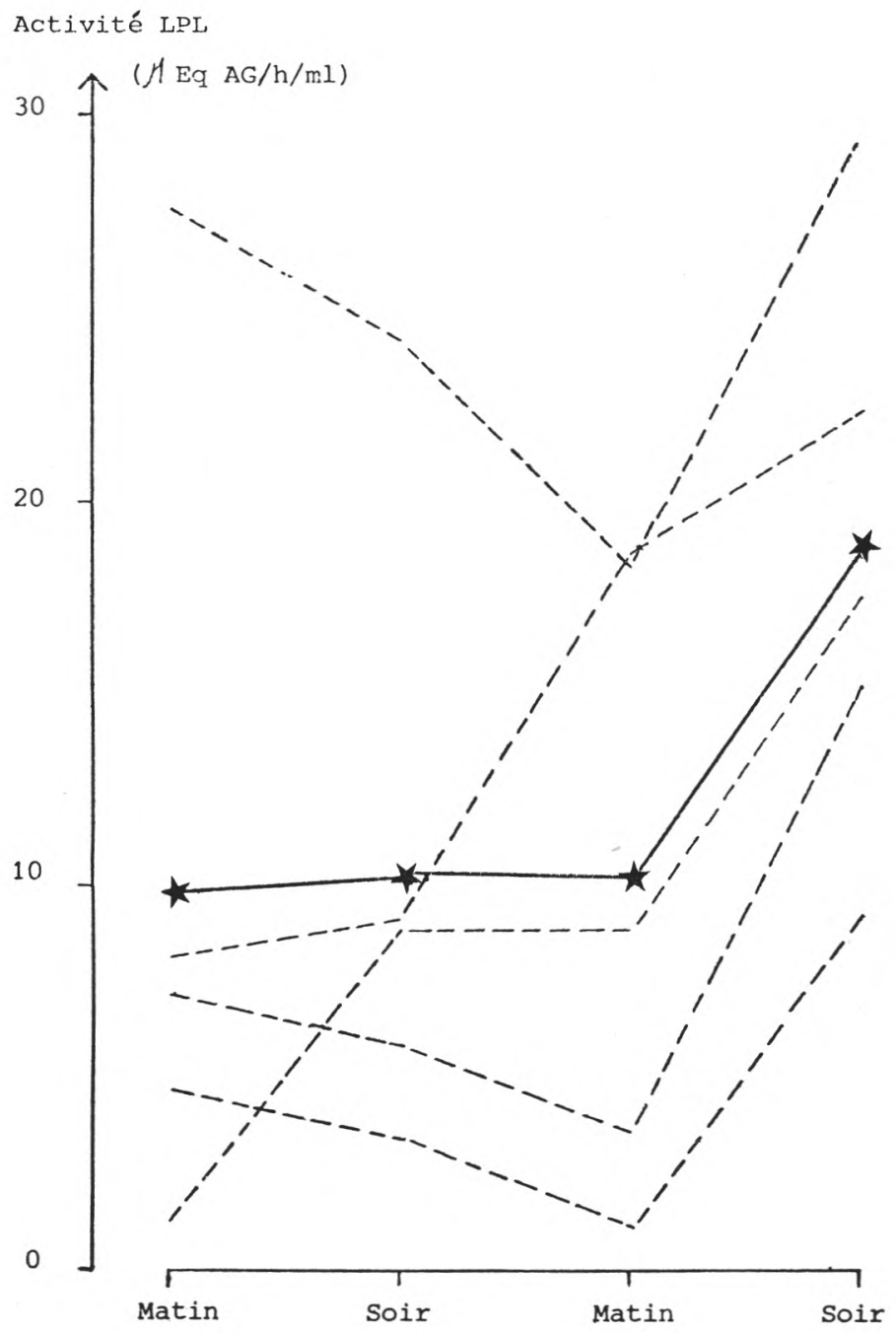

fig. 1

Variations de l'activité LPL du lait au cours de quatre traites consécutives

Le lait est prélevé manuellement au cours de quatre traites consécutives, chez cinq chèvres. Les effets jour, heure, interaction jour-heure, et chèvre sont significatifs au seuil $1 \mathrm{p}$. 1000. La dispersion des résultats se décompose comme suit pour les différents effets : jour, 6,6 p. 100 ; heure, 7,0 p. 100 ; interaction, 5,6 p. 100 ; chèvre, 67,8 p. 100 ; double d'incubation, 0,1 p. 100 ; résiduel, 12,9 p. 100 . Les étoiles $(\star)$ représentent la moyenne de chaque prélèvement. 


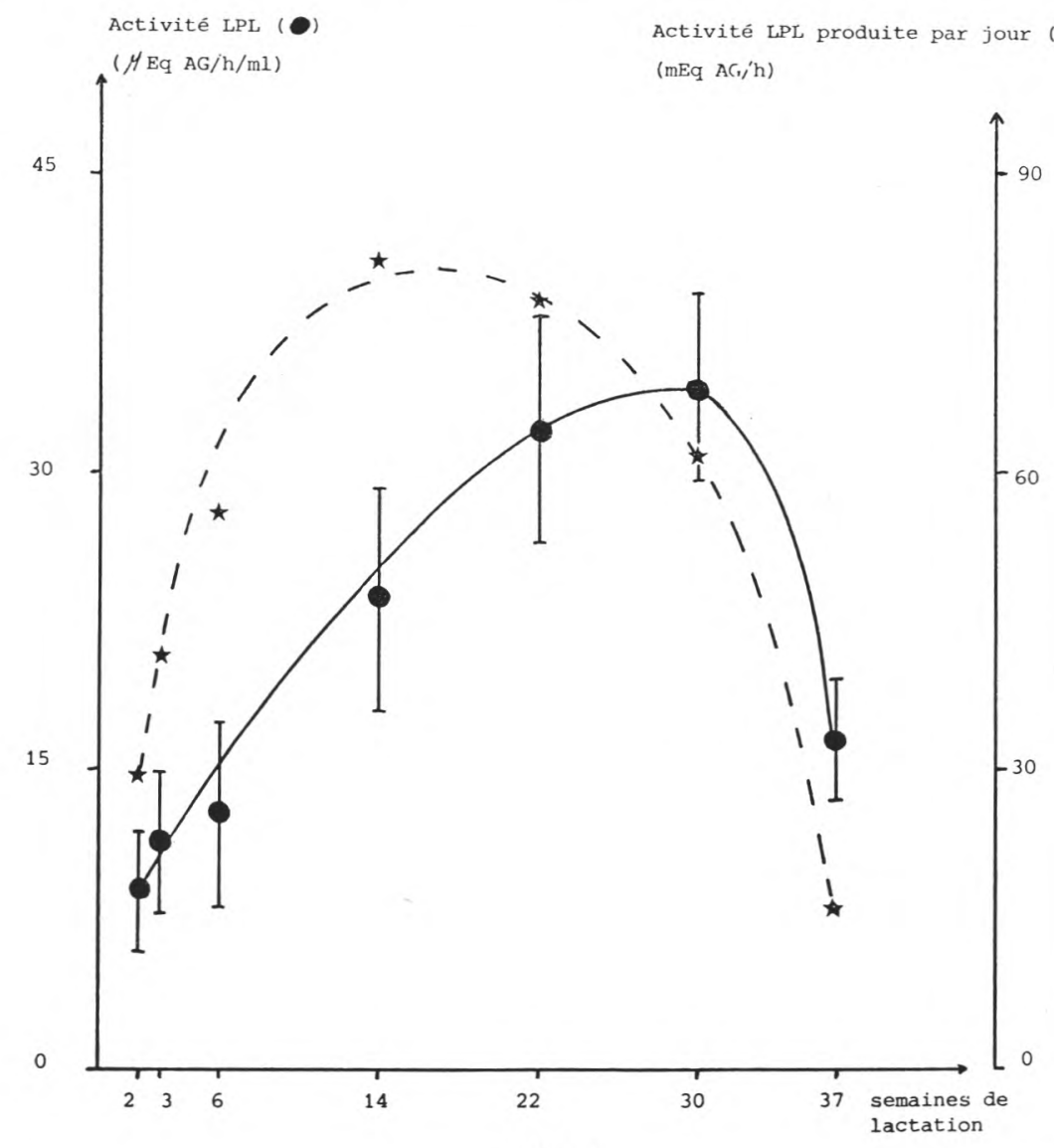

fig. 2

Evolution de l'activité LPL du lait au cours de la lactation

La moyenne ( \pm erreur-type) des résultats obtenus chez six chèvres est représentée. Les données manquent pour une chèvre en $6^{\mathrm{e}}$ et $14^{\mathrm{e}}$ semaine. La régression linéaire de l'activité sur le temps explique 51 p. 100 de la dispersion des données, de la $2^{\mathrm{e}}$ à la $30^{\mathrm{e}}$ semaine de lactation, 26 p. 100 étant expliqués par l'effet chèvre. Ces deux effets sont significatifs au seuil 1 p. 1000 .

thèse que les activités sont égales le matin et le soir) est maximum dès la $14^{\mathrm{e}}$ semaine (fig. 2).

Une corrélation " interpériode » négative et significative ( $\mathrm{R}=$ $-0,82 ; \quad \mathrm{P}<0,02)$ existe entre l'activité $\mathrm{LPL}$ et le taux butyreux du lait, la corrélation " interchèvre » étant peu significative $(\mathrm{R}=-0,64$; 


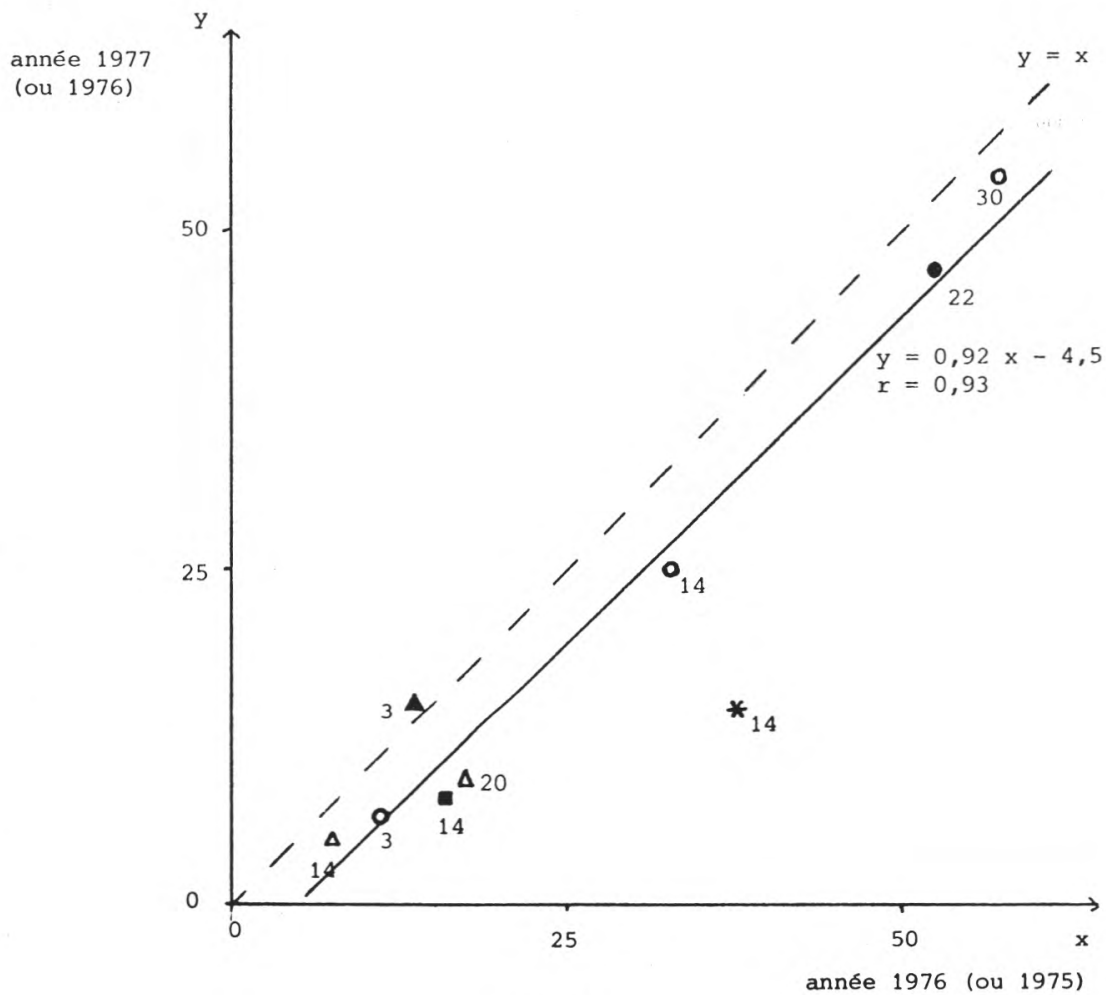

fig. 3

Activité LPL du lait mesurée 2 années successives (à stade de lactation identique)

Chaque symbole représente un animal, accompagné d'un nombre indiquant la semaine de lactation où ont été effectuées les mesures. Les coefficients de la droite de régression ne sont pas significativement différents de $1(0,92)$ et de $0(-4,5)$. Le coefficient de corrélation est significatif au seuil 1 p. 1000 .

$\mathbf{P} \simeq 0,10$ ). Les corrélations «interpériode » et « interchèvre » entre l'activité LPL et la production laitière sont négatives mais non significatives $(\mathrm{P}>0,10)$.

\section{Variations d'une lactation à la suivante}

L'activité LPL a été mesurée 2 années consécutives chez sept chèvres à des stades de lactation identiques. La figure 3 fait apparaître une certaine stabilité de l'activité LPL d'une lactation à la suivante. 


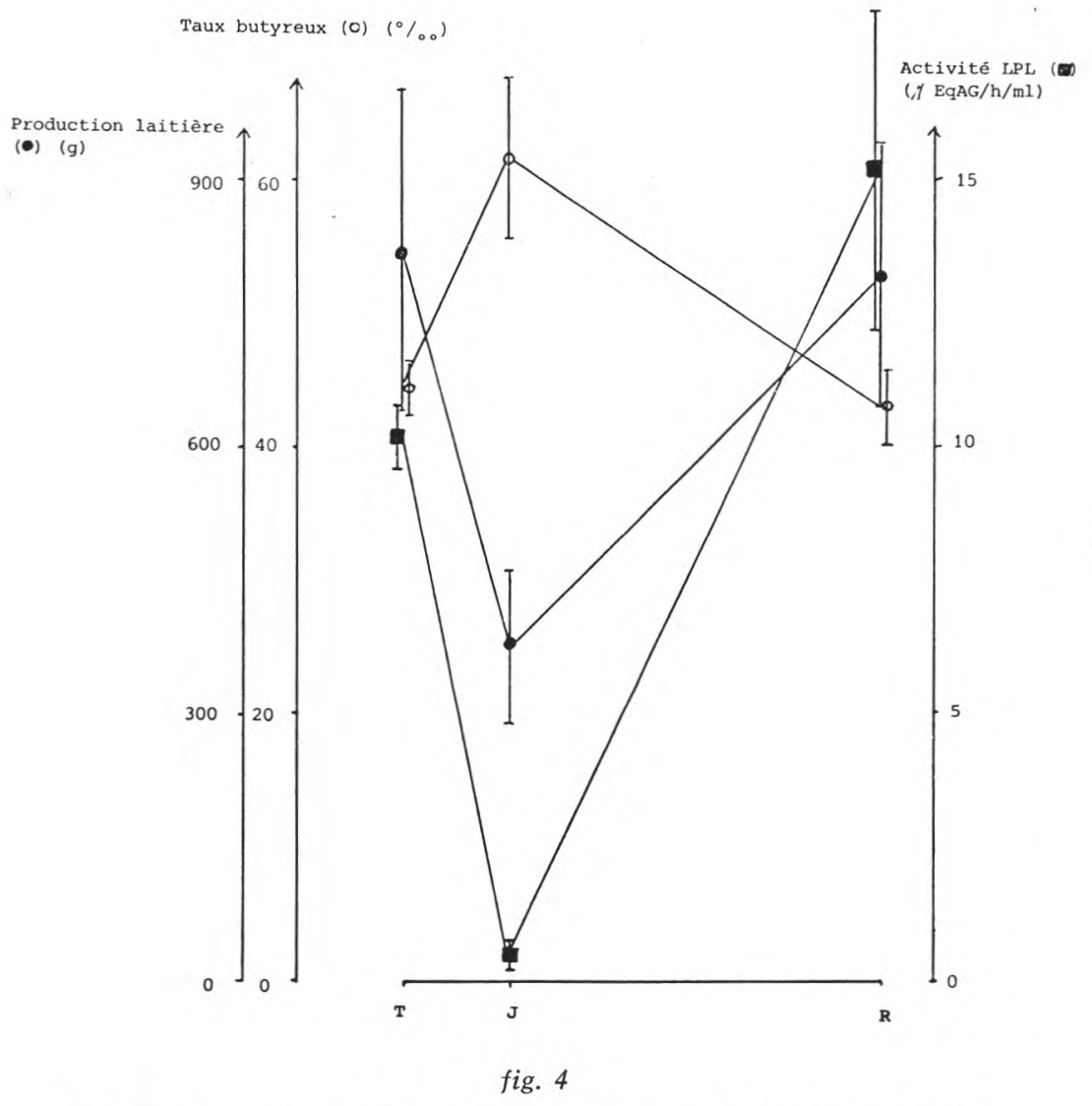

Influence du jeûne et de la réalimentation sur la production laitière, le taux butyreux et l'activité LPL du lait

Le lait est prélevé chez trois chèvres, à la traite du soir, avant la mise à jeun $(\mathrm{T}), 48 \mathrm{~h}$ plus tard $(\mathrm{J})$ et après $7 \mathrm{j}$ de réalimentation $(\mathrm{R})$. La moyenne ( \pm erreur-type) des résultats est représentée.

\section{Variations au cours du jeûne et de la réalimentation}

$\mathrm{Au}$ cours de leur $20^{\mathrm{e}}$ semaine de lactation, trois chèvres sont privées de nourriture pendant $48 \mathrm{~h}$ puis réalimentées progressivement pour atteindre en $3 \mathrm{j}$ le niveau alimentaire antérieur au jeûne. Les échantillons de lait sont prélevés le soir, juste avant la mise à jeun $(\mathrm{T})$, à la fin du jeûne $(\mathrm{J})$ et après $7 \mathrm{j}$ de réalimentation $(\mathrm{R})$.

Chez les trois chèvres, l'activité LPL (par ml de lait) diminue fortement après $48 \mathrm{~h}$ de jeûne (fig. 4 ), de même que la production 
laitière. Il y a donc une chute brutale de la quantité d'enzymes produite par traite. Le taux butyreux augmente au cours du jeûne, si bien que la quantité de matière grasse produite ne diminue que faiblement.

Après $7 \mathrm{j}$ de réalimentation, le taux butyreux et la production laitière reviennent au niveau antérieur, alors que l'activité LPL est plus élevée ( 50 p. 100 en moyenne) qu'avant le jeûne. Toutefois, les taux d'augmentation par rapport au lait témoin sont de 0-50 et 100 p. 100 pour les trois chèvres, respectivement.

\section{DISCUSSION}

\section{Teneur en LPL du lait de chèvre}

Les valeurs absolues des activités LPL du lait de chèvre de race Alpine que nous enregistrons, variant entre 0 et $70 \mu \mathrm{Eq} \mathrm{AG/h} / \mathrm{ml}$, sont inférieures à celles rapportées par Bjørke et Castberg (1976) en Norvège ( 0 à $150 \mu \mathrm{Eq} \mathrm{AG} / \mathrm{h} / \mathrm{ml})$. Ceci pourrait s'expliquer en partie par le fait que ces auteurs utilisent du sérum humain alors que nous employons du sérum sanguin de chèvre pour activer le substrat lors de la mesure de l'activité LPL du lait (ainsi que des tissus adipeux et mammaire de cette espèce, puisque la fraction physiologiquement active de l'enzyme est située sur l'endothélium des capillaires sanguins, où elle agit sur les lipoprotéines circulantes (Olivecrona et al., 1977). En effet, Brumby (1971) rapporte que les lipides plasmatiques des bovins pourraient avoir un effet inhibiteur sur l'activité LPL du lait de vache. De plus, Super et al. (1976) observent que l'ingestion par les vaches d'un régime enrichi en graisses augmente la capacité de leur sérum sanguin à activer la LPL du lait de vache. Les co-facteurs protéiques des lipoprotéines sériques (Havel et al., 1973) pourraient aussi varier avec l'espèce animale.

Les activités LPL enregistrées chez la chèvre sont inférieures à celles rapportées dans le lait de vache (jusqu'à $400 \mu \mathrm{Eq} \mathrm{AG/h/ml)}$ (Askew et al., 1970 ; Rao et al., 1973 ; Castberg et al., 1975) et de cobaye (500 à plus de $3000 \mu \mathrm{Eq} \mathrm{AG} / \mathrm{h} / \mathrm{ml}$ ) (Mc Bride et Korn, 1963 ; Hamosh et Scow, 1971). L'activité LPL du lait de femme (Luzeau et al., 1975) est voisine de celle du lait de chèvre, bien que certains prélèvements puissent atteindre les valeurs observées chez la vache (Hernell et Olivecrona, 1974 a ; Castberg et Hernell, 1975). Par contre, le lait de rate a une très faible activité LPL (environ $6 \mu \mathrm{Eq} \mathrm{AG/h/ml).}$ Ces différences pourraient être dues en partie à des variations selon les espèces dans les processus cytologiques de la sécrétion lactée (Hamosh et Scow, 1971). On peut en effet faire l'hypothèse que les cellules sécrétrices mammaires synthétisent l'enzyme LPL dont une fraction migre vers les capillaires sanguins (Olivecrona et al., 1975, 
1977), alors qu'une autre partie de l'enzyme, présente dans les cellules épithéliales mammaires en tant que " pro-enzyme ", "fuit » dans le lait au moment de la rupture de la partie apicale de ces cellules (qui est peu importante chez la rate) (Korn, 1962 ; Hamosh et Scow, 1971).

\section{Variations individuelles}

L'importance des variations individuelles de l'activité LPL du lait observée ici est générale chez les différentes espèces étudiées : chèvre (Bjørke et Castberg, 1976), vache (Askew et al., 1970 ; Castberg et al., 1975), femme (Hernell et Olivecrona, 1974 a ; Castberg et Hernell, 1975 ; Luzeau et al., 1975 ; Odièvre et Luzeau, 1976).

La relative constance de l'activité LPL du lait de chèvre, d'une lactation à l'autre, si elle était confirmée chez de nombreux animaux, pourrait permettre une caractérisation précoce des chèvres pour ce critère et faciliter des investigations sur une éventuelle origine génétique des variations individuelles observées. Deeth et Fitz-Gerald (1976) suggèrent que la susceptibilité du lait à la lipolyse pourrait être héritable, chez la vache.

\section{Variations avec le stade de traite}

Dans une précédente étude (Chilliard et Fehr, 1976 b), les activités LPL observées dans des laits de début et de fin de traite, prélevés sur deux chèvres en $22^{\mathrm{e}}$ semaine de lactation, n'étaient pas significativement différentes. Ce résultat est en accord avec ceux de Bjørke et Castberg (1976) mais pas avec ceux de Antila et AliYrkko (1975) qui rapportent une plus forte activité lipasique dans le lait de vache en début de traite. Les résultats obtenus ici ne confirment pas les précédents, pour quatre chèvres sur les cinq étudiées. Il faut toutefois noter que la mesure de 1976 portait sur $250 \mathrm{ml}$ en début et fin de traite, contre $100 \mathrm{ml}$ dans la présente étude. Il est donc probable que des variations de la teneur du lait en LPL au cours de la traite n'existent qu'aux stades extrêmes de la traite (ou qu'à l'un des deux stades seulement).

\section{Variations d'une traite à l'autre}

Les variations observées entre les traites successives ne permettent pas de conclure, contrairement à Bjørke et Castberg (1976), à une activité LPL plus élevée dans le lait du soir. En effet, dans une première expérience, les deux chèvres étudiées ont des évolutions opposées, et dans une deuxième expérience les résultats obtenus le $2^{\mathrm{e}}$ jour contredisent ceux obtenus le $1^{\text {er }}$ jour. Chez la bufflesse, Sammanwar et Ganguli (1974) rapportent une plus forte activité lipasique dans le lait du matin. Des variations journalières considérables 
de l'activité lipasique du lait ont été rapportées chez la femme (Hernell et Olivecrona, 1974 a) et la vache (Borges, 1975), et des variations hebdomadaires importantes sont observées chez la chèvre (Bjørke et Castberg, 1976 ; Chilliard et Fehr, 1976 b). Une bonne connaissance des variations liées à la traite nécessiterait de ce fait des expériences supplémentaires, portant sur un grand nombre d'animaux et de prélèvements successifs.

\section{Variations au cours de la lactation}

Les variations de l'activité LPL (ou lipasique) du lait au cours de la lactation des différentes espèces sont peu connues. Chez le cobaye, le maximum semble être atteint après 1 semaine de lactation et l'activité LPL du lait tend à varier dans le même sens que celle du tissu mammaire (Mc Bride et Korn, 1963). Chez la femme, un maximum est observé en $3^{\text {e }}$ semaine de lactation, avec de grandes variations individuelles (Odièvre et Luzeau, 1976). Une augmentation régulière de l'activité lipasique du lait est observée chez la bufflesse après la parturition (Sammanwar et Ganguli, 1974), jusqu'au $8^{\mathrm{e}}$ mois de lactation au moins (Hofi et al., 1976). Alors que Hemingway et al. (1970) et Antila et Ali-Yrkko (1975) n'ont pas observé de variations de l'activité lipasique du lait au cours de la lactation chez la vache, Dunkley et Smith (1951) rapportent une augmentation de l'activité tributyrinasique (non dissociable de l'activité LPL, Castberg et al., 1975) jusqu'en $16^{\mathrm{c}}$ semaine environ, suivie d'une chute après la $32^{\mathrm{e}}$ semaine.

Chez la chèvre, Bjørke et Castberg (1977) enregistrent, sur huit animaux une augmentation très rapide de l'activité LPL moyenne du lait entre la $4^{\mathrm{e}}$ et la $8^{\mathrm{e}}$ semaine de lactation, suivie d'une décroissance très lente jusqu'en $22^{\mathrm{e}}$ semaine. Dans cette expérience, nous observons une croissance régulière et significative de l'activité LPL du lait jusqu'à un stade beaucoup plus avancé de la lactation (22e-30 semaine).

L'activité LPL du tissu mammaire de chèvre n'ayant pas été étudiée au cours de la lactation, il n'est pas possible de tenter une comparaison entre les évolutions de cette activité et de celle du lait. Chez la rate, l'activité LPL mammaire augmente au moins jusqu'au $15^{\mathrm{e}}$ jour de lactation (Hamosh et al., 1970). Chez la vache, Shirley (1973) rapporte un maximum de cette activité en $16^{\mathrm{e}}$ semaine, avec une évolution au cours de la lactation qui se rapproche de celle observée ici pour l'activité LPL totale, produite par jour, dans le lait. Comme chez la vache (Askew et al., 1970) l'activité LPL du lait de chèvre n'est pas liée positivement au taux butyreux.

Il serait intéressant d'étudier les variations de la lipolyse spontanée dans le lait de chèvre au cours de la lactation, puisque chez la vache celle-ci augmente fortement en fin de lactation (Jellema et 
Schipper, 1975 ; Deeth et Fitz-Gerald, 1976), alors que l'activité LPL du lait des chèvres étudiées ici diminue fortement à la fin de la lactation.

\section{Variations au cours du jeûne et de la réalimentation}

La forte diminution de l'activité LPL du lait de chèvre après $48 \mathrm{~h}$ de jeûne et la restauration de l'activité pendant la période de réalimentation confirment un résultat que nous avions déjà enregistré chez une autre chèvre (Chilliard et Fehr, 1976 b).

Alors que la lipolyse spontanée augmente dans le lait des vaches sous-alimentées (Jellema et Schipper, 1975 ; Deeth et Fitz-Gerald, 1976), Borges (1975) rapporte une baisse de l'activité lipasique du lait chez la vache lors de la sous-alimentation. Toutefois, Mc Bride et Korn (1963) n'ont pas observé de baisse de l'activité LPL du lait chez un cobaye à jeun.

Les variations de l'activité LPL du lait au cours du jeûne et de la réalimentation sont parallèles à celles de l'activité LPL du tissu adipeux (épiploon), observées chez une chèvre en lactation, alors que l'activité LPL du tissu mammaire semble varier faiblement (Chilliard, 1976). Ceci pourrait être un argument en faveur de l'hypothèse de Jensen et Pitas (1976) sur une origine tissulaire extramammaire de l'activité LPL du lait (puisqu'une partie de la fraction physiologiquement active des LPL tissulaires, située sur l'endothélium des capillaires sanguins, est libérée dans le sang veineux (Robinson, 1963 ; Barry et al., 1963).

Une autre hypothèse est que la LPL du lait provienne bien de la LPL synthétisée dans les cellules sécrétrices mammaires, mais que le passage de celle-ci dans le lait soit réglé par d'autres facteurs que la quantité ou l'activité de la LPL totale (intra et extracellulaire) présente dans le tissu mammaire. La LPL du lait pourrait alors refléter l'intensité de rupture cellulaire lors de la sécrétion lactée, ou bien l'activité d'un pool intracellulaire de la LPL...

\section{CONCLUSION}

L'activité LPL du lait de chèvre varie beaucoup d'un animal à l'autre, au cours de la lactation et avec le changement d'état nutritionnel à court terme. Le lait de fin de traite tend à être plus riche en LPL que celui de début de traite. Les résultats ne permettent pas de conclure à une différence systématique entre les activités LPL du lait du matin et du soir. L'amplitude des variations observées permet de mieux prévoir le nombre d'animaux et de prélèvements pour chaque animal ainsi que les conditions de prélèvement à mettre en 
œuvre dans des expériences sur l'activité LPL du lait. L'investigation simultanée des variations de la lipolyse spontanée dans le lait de chèvre pourrait apporter des précisions sur le rôle joué dans ce phénomène par la LPL du lait de cette espèce. La mesure de l'activité LPL du lait pourrait aussi présenter un intérêt en physiologie animale, en liaison avec l'étude du métabolisme mammaire ou de la cytologie de la sécrétion lactée.

\section{Remerciements}

Nous remercions $M$. D. Sauvant qui a bien voulu relire le manuscrit, ainsi que MM. J. Hervieu et A. Cucci pour les soins donnés aux animaux et le prélèvement des échantillons de lait.

\section{Rés u mé}

Plusieurs facteurs de variation de l'activité lipoprotéine-lipasique (LPL) du lait de chèvre ont été étudiés.

L'activité LPL est plus élevée en fin de traite qu'en début de traite ; elle augmente régulièrement entre la $2^{\mathrm{e}}$ et la $30^{\mathrm{e}}$ semaine de lactation, puis chute entre la $30^{\mathrm{e}}$ et la $37^{\mathrm{e}}$ semaine et semble être stable d'une lactation à la suivante, pour un même stade de lactation. Après $48 \mathrm{~h}$ de jeûne, l'activité LPL du lait est beaucoup plus faible que chez l'animal alimenté ou réalimenté. Les variations individuelles sont importantes. Les résultats ne permettent pas de conclure à une différence systématique entre les activités LPL du lait du matin et du soir.

La mesure des variations de l'activité LPL du lait pourrait contribuer à l'étude de la lipolyse "spontanée " dans le lait ainsi que du métabolisme mammaire ou des mécanismes de la sécrétion lactée.

\section{S u m m a r y}

PHYSIOLOGICAL VARIATIONS OF GOAT MILK LIPOPROTEIN LIPASE ACTIVITY

Several factors of variation of goat milk lipoprotein lipase (LPL) activity were studied.

LPL activity was higher at the end than at the beginning of milking; it increased steadily between the 2nd and the 30th week postpartum, sharply decreased between the 30 th and the 37 th week and seemed to be stable from one lactation to the following one, and for the same lactation stage. Milk LPL activity was markedly reduced 
in 48 hours fasted goats as compared to fed or refed animals. Individual variations were important. The data did not show a systematic difference between morning and evening milk LPL activity.

The measure of milk LPL activity variations could contribute to studies on "spontaneous 》 lipolysis in milk and on mammary metabolism or milk secretion mechanisms.

Reçu pour publication en août 1977.

\section{Références bibliographiques}

ANTILA (M.) and ALI-YRKKo (S.) (1975). - Milk lipase activity. In «Proceedings of the lipolysis symposium - Cork». Fed. Int. Laiterie. Document $\mathrm{n}^{\circ}$ 86, 58-60.

ANTONIS (A.) (1965). - Semiautomated method for the colorimetric determination of plasma free fatty acids. J. Lipid Res., 6, 307-312.

Askew (E. W.), Emery (R. S.) and Thomas (J. W.) (1970). - Lipoprotein lipase of the bovine mammary gland. J. Dairy Sci., 53, 1415-1423.

BARRY (J. M.), Bartley (W.) Linzell (J. L.) and RoBinson (D. S.) (1963). - The uptake from the blood of triglyceride fatty acids of chylomicra and low density lipoproteins by the mammary gland of the goat. Biochem. J., 89, 6-11.

BJøRKe (K.) and CAstBeRg (H. B.) (1976). - Lipolytic activity in goat's milk. Nordeurop. Mejeri-Tidsskrift, 8, 296-304.

BJøRKE (K.) and CAstberg (H. B.) (1977). - Communication personnelle.

Borges (M. S.) (1975). - Milk flavors, lipase activity, and milk production as related to suden changes of energy in the cow's ration. Dissert. Abstr. Int., 35, 4733-B.

BRUMBY (P. E.) (1971). - Influence of plasma lipids on the activity of lipoprotein lipase. Biochem. J., 122, 10 P.

Castberg (H. B.), Egelrud (T.), Solberg (P.) and Olivecrona (T.) (1975). - Lipases in bovine milk and the relationship between the lipoprotein lipase and tributyrate hydrolysing activities in cream and skim-milk. J. Dairy Res., 42, 255-266.

Castberg (H. B.) and Hernell (O.) (1975). - Role of serum-stimulated lipase in lipolysis in human milk. Milchwissenschaft, 30, 721-724.

Castberg (H. B.) and Solberg (P.) (1974). - The liproprotein lipase and the lipolysis in bovine milk.Meieriposten, 63 (61/52), 961.

Chilliard (Y.) (1976). - Résultats non publiés.

Chilliard (Y.) et Fehr (P. M.) (1976 a). - Activité lipolytique du lait de chèvre. I. Mise en évidence d'une activité lipoprotéine-lipasique. Ann. Techn. agric., $25,219-230$.

Chilliard (Y.) et Fehr (P. M.) (1976 b). - Activité lipolytique du lait de chèvre. II. Quelques variations physiologiques de l'activité lipoprotéine-lipasique. Ann. Techn, agric., 25, 231-237.

Chilliard (Y.) et Luzeau (R.) (1975). - Résultats non publiés.

DeEth (H. C.) and Fitz-Gerald (C. H.) (1975), - Factors governing the susceptibility of milk to spontaneous lipolysis. In "Proceeding of the lipolysis symposium - Cork». Fed. Int. Laiterie. Document $n^{\circ}$ 86, 24-34.

DeEth (H. C.) and Fitz-Gerald (C. H.) (1976). - Lipolysis in dairy products: a review. Austr. J. Dairy Technol., 31, 53-64. 
DunKLeY (W. L.) and Smith (L. M.) (1951). - Hydrolytic rancidity in milk. IV. Relation between tributyrinase and lipolysis. J. Dairy Sci., 34, 940-947.

DRIESSEN (F. M.) and STADHOUdERS (J.) (1974). - A study of spontaneous rancidity. Neth. Milk Dairy J., 28, 130-145.

Egelrud (T.) and Olivecrona (T.) (1972). - The purification of a lipoprotein lipase from bovine skim milk. J. Biol. Chem., 247, 6212-6217.

FÉdÉRATION INTERNATIONALE DE LAITERIE (1974). — La lipolyse dans le lait refroidi en vrac. Document $\mathrm{n}^{\circ} 82$.

Fédération InTERnationale de laiterie (1975). - Proceedings of the lipolysis symposium - Cork (Ireland), 5-7 march 1975, compiled by W. K. DownEY and J. M. Cogan. Document $\mathrm{n}^{\circ} 86$.

Hamosh (M.), Clary (T. R.), Chernick (S. S.) and Scow (R. O.) (1970). - Lipoprotein lipase activity of adipose and mammary tissue and plasma triglyceride in pregnant and lactating rats. Biochim. Biophys. Acta, 210, 473-482.

Hamosh (M.) and Scow (R. O.) (1971). - Lipoprotein lipase activity in guinea pig and rat milk. Biochim. Biophys. Acta, 231, 283-289.

Havel (R. J.), Fielding (C. J.), Olivecrona (T.), Shore (V. G.), Fielding (P. E.) and EGELRUD (T.) (1973). - Cofactor activity of protein components of human very low density lipoproteins in the hydrolysis of triglycerides by lipoprotein lipase from different sources. Biochemistry, 12, 1828-1833.

Hemingway (E. B.), Smith (G. H.), Rook (J. A. F.) and O'Flanagan (N. C.) (1970). Lipase taint. J. Soc. Dairy Techn., 23, 44-48.

Hernell (O.) and Olivecrona (T.) (1974 a). - Human milk lipases. I. Serumstimulated lipase. J. Lipid Res., 15, 367-374.

Hernell (O.) and Olivecrona (T.) (1974 b). - Human milk lipases. II. Bile saltstimulated lipase. Biochim. Biophys, Acta, 369, 234-244.

Hofi (A. A.), Mahran (G. A.), Abdel-Hamid (L. B.) and Osman (S. G.) (1976). Lipase activity in buffaloes'milk. I. Effect of lactation period and individuality. Egypt. I. Dairy Sci., 4, 111-114 (in Dairy Sci. Abstr., 39, (1977), abstr. $\mathrm{n}^{\circ} 2700$ ).

Hoynes (M. C. T.) and Downey (W. K.) (1973). - Relationship of the lipase and lipoprotein lipase activities of bovine milk. Biochem. Soc. Transact., 1, 256-259.

JELlema (A.) and SchIPPER (C. J.) (1975). - Influence of physiological factors on the lipolytic susceptibility of milk. In "Proceedings of the lipolysis symposium - Cork» Fed. Int. Laiterie. Document $n^{\circ}$ 86, 2-6.

Jensen (R. G.) and Pitas (E.) (1976). - Milk lipoprotein lipases: a review. J. Dairy Sci., 59, 1203-1214.

KORN (E. D.) (1962). - The lipoprotein lipase of cow's milk. J. Lipid Res., 3, 246-250.

Luzeau (R.), Odièvre (M.), Levillain (P.) et Lemonnier (A.) (1975). - Activité de la lipoprotéine-lipase dans les laits de femme inhibiteurs in vitro de la conjugaison de la bilirubine. Clin. Chim. Acta, 59, 133-138.

Mc BRIDE (O. W.) and KORN (E. D.) (1963). - The lipoprotein lipase of mammary gland and the correlation of its activity to lactation. J. Lipid. Res., 4, 17-23.

Odièvre (M.) et Luzeau (R.) (1976). - Communication personnelle.

Olivecrona (T.), Egelrui (T.), Hernell (O.), Castberg (H.) and Solberg (P.) (1975). - Is there more than one lipase in bovine milk ? In "Proceeding of the lipolysis symposium - Cork». Fed. Int. Laiterie. Document $n^{\circ}$ 86, 61-72.

Olivecrona (T.), Bengtsson (G.), Marklund (S. E.), Lindhal (K.) and Hook (M.) (1977). - Heparin-lipoprotein lipase interactions. Fedn. Proc., 36, 60-64. 
RaO (D. R.), HaWkins (G. E.) and SMith (R. C.) (1973). - Effect of glucose and insulin on lipoprotein lipase activity in adipose tissue and milk. J. Dairy Sci., 56, 1415-1419.

RoBINSON (D. S.) (1963). - Clearing factor lipase and its action in the transport of fatty acids between the blood and the tissues. Adv. Lipid Res., 1, 133-181.

Sammanwar (R. D.) et Ganguli (N. C.) (1974). - La lipase du lait de bufflesse. $19^{e}$ Congrès Int. Laiterie, New-Dehli, 1 F, 380.

SHIRLEY (J. E.) (1973). - Regulatory aspects of triglyceride uptake by bovine adipose and mammary tissues. Ph. D. thesis, Michigan State Univ., East Lansing (U.S.A.).

Super (D. M.), Palmouist (D. L.) and Schanbacher (F. L.) (1976). - Relative activation of milk lipoprotein lipase by serum of cows fed varying amounts of fat. J. Dairy Sci., 59, 1409-1413.

Tarassuk (N.P.) and Frankel (E. N.) (1957). - The specificity of milk lipase. IV. Partition of the lipase system in milk. J. Dairy Sci., 40, 418-430. 